\title{
Performance assessment of neuro fuzzy based image fusion of satellite images
}

\author{
Ch. Ramesh Babu ${ }^{1 *}$, D. Srinivasa Rao $^{2}$, T. Ravi $^{2}$ and G. Gopi ${ }^{3}$ \\ Professor, Department of Computer Science, GCET, Hyderabad, India ${ }^{1}$ \\ Associate Professor, Department of IT, VNRVJIET, Hyderabad, India ${ }^{2}$ \\ Assistant Professor, Department of Computer Science, Akamai Tech, Hyderabad, India ${ }^{3}$
}

(C2018 ACCENTS

\begin{abstract}
Image fusion is a technique to converge multispectral (MS) and panchromatic (PAN) images in to a one fused image which is moderately supplementary helpful compared to input images taken for fusion. Image fusion is an important task to recover an image which delivers as much as evidence of the same body part at the similar time it also assistances to decrease the storing capability to a particular image. In this paper an assessment is completed among conventional image fusion methods; principal component analysis (PCA), discrete wavelet transform (DWT), IHS transform based fusion, Brovey transform based fusion, and the projected neuro fuzzy based iterative image fusion techniques. The proposed neuro fuzzy based iterative fusion method utilizes fuzzy inference system (FIS) prepared by determining fuzzy rules and membership functions precisely. Experimentations have been finished on different datasets of multimodal satellite images. The projected technique is perceivably and significant related with the other fusion approaches. For the assessment of the fused image obtained from various fusion techniques ten diverse measures is prepared and utilized of, namely image quality index (IQI) and mutual information measure (MIM) with probability density.
\end{abstract}

\section{Keywords}

Image fusion, PCA, DWT, IHS, Brovey transform.

\section{Introduction}

Functions (PDF) for inputs, root mean square error (RMSE), peak signal to noise ratio(PSNR), correlation coefficient (CC) and spatial frequency (SF). Assessment outcomes demonstrated that the projected neuro fuzzy based image fusion technique improved image quality than any of the conventional image fusion techniques. Image fusion to converge evidence from source images of a same section into a one combined image that is additional useful and is added appropriate for conception or computer handling domains. A structure is planned in which combines the welfares of a fuzzy validation and a neural structure. The framework seams collected Kalman unscrambling and subtle treating recommendation i.e. ANFIS to organization an operative evidence grouping approach for the objective subsequent outline. An original multipurpose intention motivated around ANFIS is projected to regulate rational developments and to deteriorate the uncertain exacerbation of approximation evidence from multisensory.

\footnotetext{
*Author for correspondence
}

Fuzzy adaptable amalgamation scheming is a convincing device to make the genuine superiority of the excess covariance steady with its theoretical value. ANFIS designates excessive captivating in and projection abilities, which varieties it a creative device to achieve practiced susceptibilities in any outline. A neural organization is accessible, which can essence the assessable possessions of the models during the planning terms [1]. Image fusion method has been utilized in pronounced domains: medical image processing, satellite image processing, computer vision, involuntary change recognition, biometrics and armed solicitations. Multi-device image combination for investigation schemes deliberated where fuzzy method exploited for fusing images taken from various sensors, in order to improve conception for observation [2]. The source images decomposition by wavelet transform three consistency structures are mined and then a fuzzy instruction is utilized to combine wavelet factors from the two images conferring to the mined structures. Image fusion procedure built on fuzzy approach and wavelet transform, motivated on observable and electromagnetic image fusion and discourse a procedure centered on the DWT and fuzzy approach [3] and the method formed two fuzzy 
associations and assessed the standing of each wavelet factor with fuzzy perception. A fuzzy and neuro-fuzzy technique projected for image fusion and later exploits the image fusion procedure in iterative approach by means of fuzzy and neuro fuzzy method. [4]. A different method to the medical image fusion, engaging geometric examination of the non-sub sampled contourlet transform and fuzzy-adaptive reduced pulse-coupled neural network). Practice of the reduced pulse-coupled neural network (RPCNN) with a smaller amount of complex structure and having less number of parameters leads to computational efficiency and significant condition of point-of-care strength care approaches. The projected arrangement is allowed from the collective failings of the recent approaches [5]. Projected a Neuro-fuzzy technique of image fusion eliminates the spatial falsehood of wavelet based image fusion technique and incorrect boundaries as well as shady adverts [6].

\section{Principal component analysis based image fusion}

2.1PCA based image fusion

The MS and PAN images signified by $\mathrm{M}_{1}(\mathrm{x}, \mathrm{y})$ and $\mathrm{M}_{2}$ ( $\mathrm{x}, \mathrm{y}$ ) and transform these inputs in to corresponding two column directions and deduct their means. The quantity of the productivity vector is $\mathrm{n} \times 2$, where $\mathrm{n}$ is the size of the image vector. The Eigen standards and conform Eigen directions for this result vector is determined and also estimate the Eigen directions associated to the greater Eigen standards. $\mathrm{C}_{1}$ and $\mathrm{C}_{2}$ are consistent components intended from covariance matrix to accomplish Eigen direction and the output fused ' $F$ ' image is attained from it $[3,7]$.

$\mathrm{F}=\mathrm{c} 1 \mathrm{~m} 1(\mathrm{x}, \mathrm{y})+\mathrm{c} 2 \mathrm{~m} 2(\mathrm{x}, \mathrm{y})$

\section{Wavelet transform based image fusion}

Discrete wavelet transform (DWT) based fusion with a various factors selected method is planned and by exploiting the DWT approach source inputs are decomposed and two disparate window-based image fusion instructions are differently applied to merge $\max$ and $\min$ frequency factors. Subsequently the min frequency field factors with more content values are chosen as elements of the output [8].

\section{IHS transform based fusion method}

The IHS transform is extensively utilized an image fusion method to make use of the complementary landscape of multisensory image information. Formerly directing an IHS fusion, the source image ought to be recorded with the great determination
PAN image and would be re-elected to the similar pixel extent with the PAN image. Subsequently, the three ensembles ( $r, g$, and $b$ ) of a source image have to be converted the RGB intergalactic addicted to the IHS intergalactic [9].

\section{Brovey transform based fusion method} The Brovey method is built to rectify the drawbacks of the multiplicative technique. It is a smooth approach of converging information collected various sensors. It is a mixture of mathematical calculations and regularize the colour channels prior to they are multiplied with the PAN image.

The equation utilized for the Brovey method would DESIGNATED as Red $=($ band $1 / \sigma$ band $n) *$ more resolution channel, Green $=($ band $2 / \sigma$ band $n) *$ more resolution channel, Blue $=($ band $3 / \sigma$ band $n) *$ more resolution channel, high resolution band $=$ PAN [10].

DWT and sparse representation (SR) based fusion approach is suggested and proved that investigational results proved that the impact of suggested approach in terms quality and visual version [4]. A multi-band image fusion approach ground on unsupervised spectral unmixing for collaborating high-dimensional and low-haunted determination image and a lowspatial high-spectral resolution image [5]. Suggested a recent $\mathrm{CNN}$-based multi-focus image fusion approach that displays the capability of CNNs for other-type image fusion subjects. Also locates advancing some proposals on the upcoming training of CNN-based image fusion [6].

\section{Neuro fuzzy based iterative image fusion}

The intention of the approach for iterative image fusion using neuro fuzzy fusion is to restate the image fusion process phases iteratively, in which output image is utilized as one of the input images from second iteration onwards. Image fusion using fuzzy logic method is combined with artificial neural networks to execute fusion with neuro fuzzy logic approach.

The input image data is utilized to assess the concert of the system. The procedure of crucial membership functions and fuzzy rules for the image fusion using fuzzy inference system (FIS) editor of fuzzy logic toolbox in Matlab. The membership functions comprise pixel values that are picked up on the basis of suspicions of the input images. The neural network toolbox benefits us to act out the qualified neural 
network based on evalfis as well as the ANFIS functions that are utilized to accomplish the fuzzy inference intentions.

Neuro fuzzy based iterative image fusion algorithm is proposed to execute fusion process on MS and PAN images. As part of executing fusion algorithm precise fuzzy rules and membership functions are determined. Soft computing techniques are utilized in this paper to enhance the fused image content. The procedure for image fusion utilizing neuro fuzzy is deliberated and similar procedure executed iteratively here [11].

- Recite MS image in i1 and catch its extent (rows: zl, columns: sl).

- Recite pan image in i2 and catch its extent (rows: z2. Columns: s2).

- I1 and i2 are images in matrix; individual pixel is in the excellent from 0-255. Use gray color map.

- Associate size of two input images. If source images are not of the related extent, choose the sections are of similar extent.

- Transform the inputs in column practice where it has $\mathrm{c}=\mathrm{zl}^{*} \mathrm{sl}$ admittances.

- Practice preparation information is a matrix with three columns.

- Practice patterned information is a matrix of pixels of two inputs are in column arrangement.

- Determine amount and category of association utilities for source images by standard the association utilities.

- FIS is utilized is obtained by genfis1 instruction with prepared information, quantity association utilities and category of association utilities as input.

- To begin preparing, ANFIS is utilized where inputs produced FIS and prepared information and proceeds prepared information.

- For num=1 to $\mathrm{c}$ in stages of single, utilize fuzzification by means of the produced FIS with check information and prepared information as inputs which proceeds fused $\mathrm{t}$ image in column format.

- Endure the fusion technique with two inputs, in which single participation is the output gained from the fusion and another one is the essential source image.

- Transform the vertical form to matrix and demonstrate the output image membership functions and fuzzy instructions are utilized in the projected neuro fuzzy based iterative image fusion method.
Axiom-1:

[input_1 is $\mathrm{mf} \_1$ ] and [input_2 is $\left.\mathrm{mf} \_2\right]$

$\rightarrow$ [output_1 is msf_1]

Axiom-2:

[input_1 $\rightarrow$ mf_2] and [input_2 is mf_2]

$\rightarrow$ [output_1 is mf_2]

Axiom-3:

[input_1 $\rightarrow$ mf_3] or [input_2 is mf_2]

$\rightarrow$ [ouput_1 is mf_3]

Axiom-4:

[input_1 $\rightarrow$ mf_1] and [input_2 is mf_3]

$\rightarrow$ [output_1 is $\mathrm{mf}_{-}$1]

Axiom-5:

[input_1 $\rightarrow$ mf_3] or [input_2 is mf_3]

$\rightarrow$ [output_1 is mf_2]

\section{Assessment metrics for fused image}

Assessment parameters are utilized to assess the excellence of the output image. The output images are assessed, enchanting the succeeding metrics into contemplation.

\subsection{Image quality index}

Image quality index (IQI) dealings with the resemblance concerning inputs (i1 \& i2) and it's worth sorts from -1 to 1 . IQI is equivalent to 1 if dual inputs are similar. IQI quantity is specified by [12].

$$
I Q I=\frac{m_{a b} 2 x y 2 m_{a} m_{b}}{m_{a} m_{b} x^{2}+y^{2} m_{a}^{2}+m_{b}{ }^{2}}
$$

Where $\mathrm{x}$ and $\mathrm{y}$ signify the mean standards of inputs i1 and i2 and $m_{a}^{2}, m_{b}^{2}$ and $m_{a b}$ signifies the variance of i1, i2 and covariance of i1 and i2.

IQI value should be high for better fused image indicates that quality of fused image is improved through fused image.

\subsection{Mutual information measure}

Mutual information measure (MIM) delivers the quantity of evidence of single input in added. It contributes the procedures for choosing the greatest fusion technique. Certainly take inputs $m(i, j)$ and $n$ $(i, j)$ and MIM concerning inputs is distinct as:

$$
I_{M N}=\sum_{x, y} P_{M N}(x, y) \log \frac{P_{M N}(x, y)}{P_{M}(x) P_{N}(y)}
$$

Where, $\mathrm{p}_{\mathrm{m}}(\mathrm{x})$ and $\mathrm{p}_{\mathrm{n}}(\mathrm{y})$ are the PDF in the individual images, and $p_{\mathrm{mn}}(x, y)$ is joint PDF. Better fused images will have high MIM values indicate that MS and PAN image information is mutually fused. 
Babu et al.

\subsection{Root mean square error}

The root mean square error (RMSE) processes the quantity of alteration each pixel caused by the dealing out. The RMSE concerning an ideal image $r$ and the output image $f$ is specified by

$$
R M S E=\sqrt{\frac{1}{M N} \sum_{i=1}^{M} \sum_{j=1}^{N}(R(i, j)-F(i, j))}
$$

RMSE value should be low for better fused image. Error value is low between reference image and fused image.

\subsection{Peak signal to noise ratio}

Peak signal to noise ratio (PSNR) can be designed by by means of the formulary

$$
P S N R=20 \log _{10}\left[\frac{L^{2}}{M S E}\right]
$$

One is the amount of gray planes in the fused image. Better fused images are having high PSNR values as reciprocal to RMSE values.

\subsection{Correlation coefficient}

The correlation coefficient (CC) technique is utilized to conclude how thoroughly the source image and fused images co-vary. $\mathrm{Cc}$ is extensively exploited aimed at associating inputs [13].

$$
C C=\frac{\sum_{i=1}^{n}(X i-X)(Y i-Y)}{\sqrt{\sum_{i=1}^{n}\left(X i-\overline{X)^{2}} \sqrt{\sum_{i=1}^{n}\left(Y i-\overline{Y)^{2}}\right.}\right.}}
$$

Where, $x_{i}$ is the strength of the $i^{\text {th }}$ pixel in input $1, y_{i}$ is the strength of the $i^{\text {th }}$ pixel in input2, $x$ is the mean strength of input 1 and $y$ is the mean strength of input2. A higher CC value for fused images indicates that fusion process is performed well. So that better correlation is formed between input image and fused image.

\subsection{Spatial frequency}

The spatial frequency (SF) in spatial state designates the complete action level in the output image [14].

$$
S F={\sqrt{(R F)^{2}+(C F)^{2}}}^{2}
$$

A higher value of spatial frequency for fused image indicates that overall activity level in the fused image is more.

\section{Experimental results and analysis}

In this segment, six case studies (CS) are taken and accomplished image fusion between MS and PAN images as shown in Figure 1.

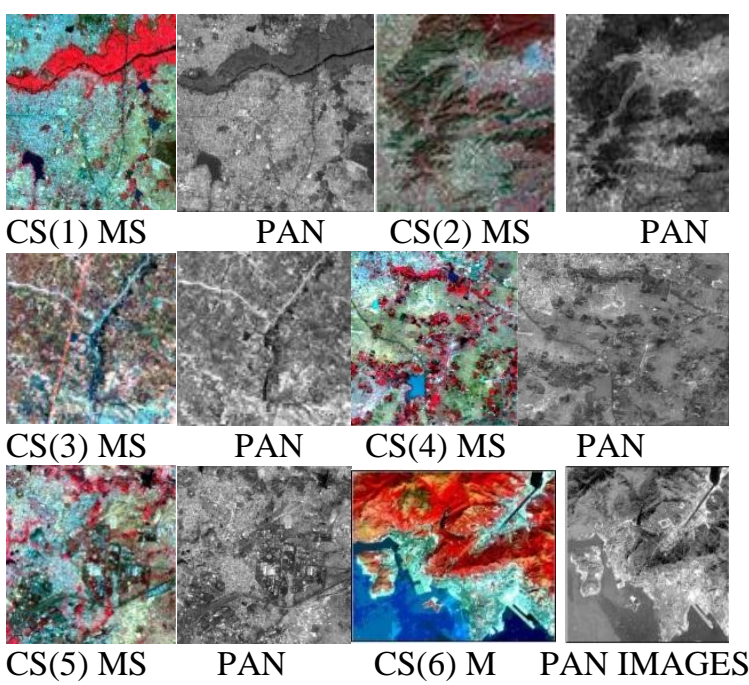

Figure 1 Six case studies of images considered for image fusion

The fusion techniques are implemented in Matlab 10.0. In order to assess the fusion outcomes attained from various approaches evaluation metrics are engaged (Figure 2). The values of respective superiority evaluation metrics of all declared image fusion methods are portrayed in Table 1 .
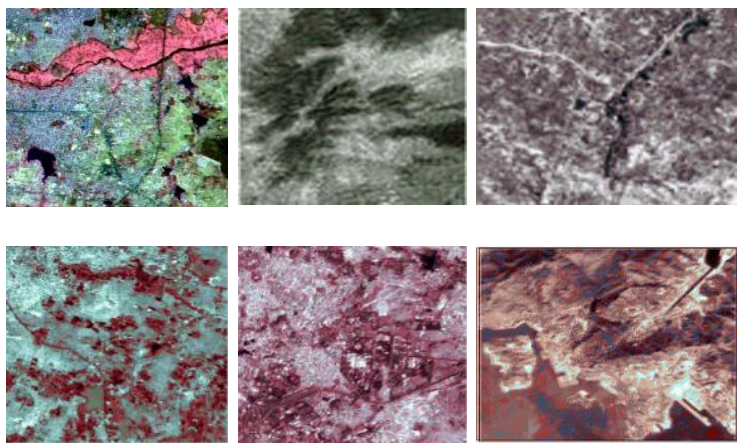

Figure 2 PCA based fused images for six case studies of images considered for fusion

Compared to MS and PAN image visualization fused image obtained through PCA based fused image visual perception is improved and statistical parameters also proved that fused image content is enriched.

DWT based fusion process improves spectral content and spatial content taken from MS and PAN images and improves visual perception and IQI, MIM, PSNR, CC and SF is also improved and proved through statistical parameters utilized for assessment fused image (Figure 3). 
Fused images obtained from IHS based image fusion process are with enriched visual quality and also having more image content than MS and PAN images (Figure 4).

Fused images obtained from Brovey transform based fusion process are more informativs and visual perception is also enriched (Figure 5).
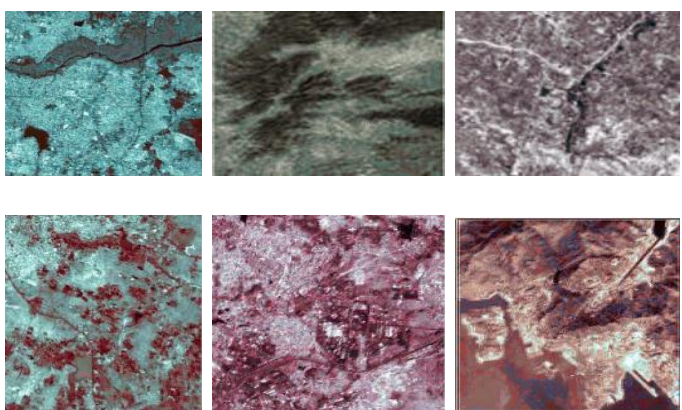

Figure 3 DWT based fused images for six case studies of images considered for fusion
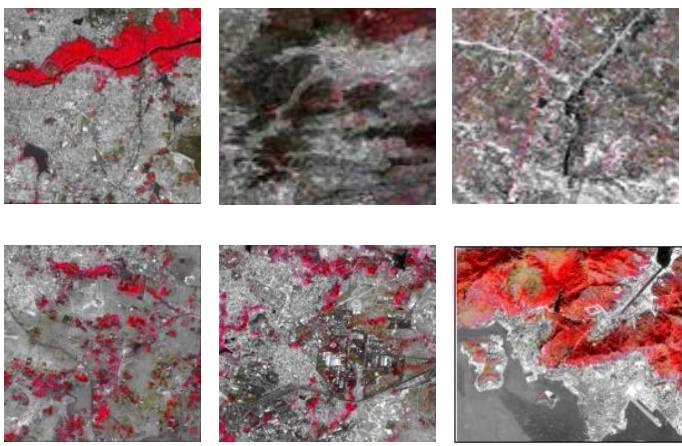

Figure 4 IHS transform based fused images for six case studies of images considered for fusion
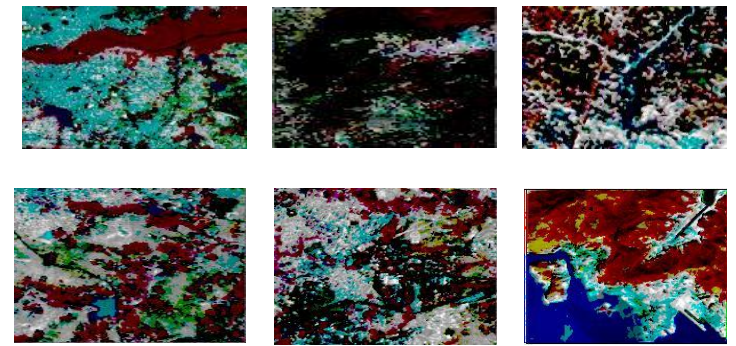

Figure 5 Brovey transform based fused images for six case studies of images considered for fusion

Neuro fuzzy based fusion process advances visual perception by extracting spectral and spatial content mutually from MS and PAN images and improves observable content and also IQI, MIM, PSNR, CC and $\mathrm{SF}$ values are improved and proved through statistical parameters utilized for assessment fused image in all datasets (Figure 6 and 7).

47
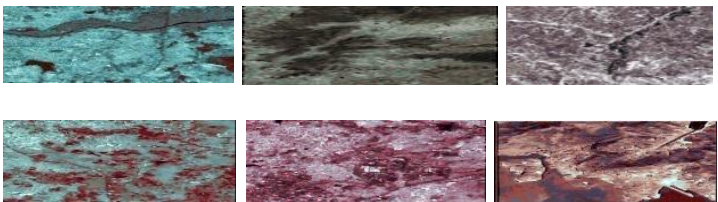

Figure 6 Neuro fuzzy based fused images for six case studies of images considered for image fusion
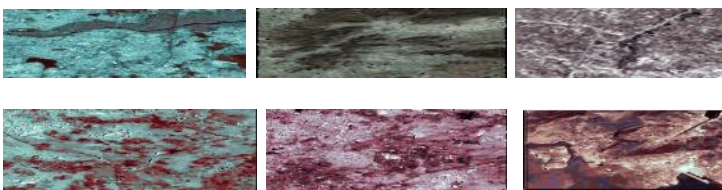

Figure 7 Neuro fuzzy based iterative fused images for six case studies of images considered for fusion

In all datasets fused image visual perception is enhanced and also shown that image quality (IQI), mutual content (spectral and spectral) taken from MS image and PAN image, peak signal to noise ratio, correlation coefficient, spatial frequency are also polished through fused image assessment parameters. All statistical parameters like IQI, MIM, PSNR, CC and SF values are more for fused image obtained from proposed neuro fuzzy based iterative fusion process and neuro fuzzy based fusion followed by DWT based fusion and PCA based fusion methods. From the observation of results illustrated in Table 1 proposed neuro fuzzy based iterative image fusion produces better results followed by neuro fuzzy based one-time image fusion and later positions are taken by conventional techniques mentioned in the paper. The strength of the neuro fuzzy based image fusion enhances the quality of the fused image and also obtained mutual information from ma and pan images into a fused image. By reducing the RMSE values and improving PSNR values for fused images obtained from proposed method designates that image content is enriched.

\section{Conclusions}

In this paper, the proposed neuro fuzzy based fusion and conventional techniques are implemented. A fused result obtained from various techniques has been discussed through assessment metrics. The investigational outcomes obviously demonstrated that the projected neuro fuzzy based iterative fusion for image fusion provides a substantial improvement in evaluation metrics. Due to potentiality of neuro fuzzy outcomes obtained from proposed method enhances the content of fused images compared to other approaches. In conclusion neuro fuzzy based iterative image fusion conserves additional quality evidence. In future it is anticipated that the proposed fusion technique can be supplementary protracted to all 
categories of images for fusion and also expected that the procedure can be supplementary protracted to video image processing and for fusion of numerous sensor inputs and to integrate valid assessment metrics of image fusion.

\section{Acknowledgment}

None.

\section{Conflicts of interest}

The authors have no conflicts of interest to declare.

\section{References}

[1] Petković D. Adaptive neuro-fuzzy fusion of sensor data. Infrared Physics \& Technology. 2014; 67:222-8.

[2] Zheng Y, Zheng P. Multisensor image fusion using fuzzy logic for surveillance systems. In international conference on fuzzy systems and knowledge discovery 2010 (pp. 588-92). IEEE.

[3] Zhu M, Yang Y. A new image fusion algorithm based on fuzzy logic. In international conference on intelligent computation technology and automation 2008 (pp. 83-6). IEEE.

[4] Ranjan R, Singh H, Meitzler T, Gerhart GR. Iterative image fusion technique using fuzzy and neuro fuzzy logic and applications. In annual meeting of the North American fuzzy information processing society 2005 (pp. 706-10). IEEE.

[5] Das S, Kundu MK. A neuro-fuzzy approach for medical image fusion. IEEE Transactions on Biomedical Engineering. 2013; 60(12):3347-53.

[6] Teng J, Wang S, Zhang J, Wang X. Neuro-fuzzy logic based fusion algorithm of medical images. In image and signal processing (CISP), 3rd international congress 2010 (pp. 1552-6). IEEE.

[7] Naidu VP, Raol JR. Pixel-level image fusion using wavelets and principal component analysis. Defence Science Journal. 2008; 58(3):338-52.

[8] Yang Y, Huang S, Gao J, Qian Z. Multi-focus image fusion using an effective discrete wavelet transform based algorithm. Measurement Science Review. 2014; 14(2):102-8.

[9] El-Mezouar MC, Taleb N, Kpalma K, Ronsin J. An IHS-based fusion for color distortion reduction and vegetation enhancement in IKONOS imagery. IEEE Transactions on Geoscience and Remote Sensing. 2011; 49(5):1590-602.

[10] Mandhare RA, Upadhyay P, Gupta S. Pixel-level image fusion using brovey transforme and wavelet transform. International Journal of Advanced Research in Electrical, Electronics and Instrumentation Engineering. 2013; 2(6):2690-5.

[11] Dammavalam SR, Maddala S, Prasad MK. Quality evaluation measures of pixel-level image fusion using fuzzy logic. In international conference on swarm, evolutionary, and memetic computing 2011 (pp. 48593). Springer, Berlin, Heidelberg.

[12] Mumtaz A, Majid A, Mumtaz A. Genetic algorithms and its application to image fusion. In international conference on emerging technologies 2008 (pp. 6-10). IEEE.

[13] Dammavalam SR, Maddala S, Prasad MK. Iterative image fusion using fuzzy logic with applications. In advances in computing and information technology 2013 (pp. 145-52). Springer, Berlin, Heidelberg.

[14] Rao DS, Seetha M, Hazarath M. Iterative image fusion using neuro fuzzy logic and applications. In international conference on machine vision and image processing 2012 (pp. 121-4). IEEE.

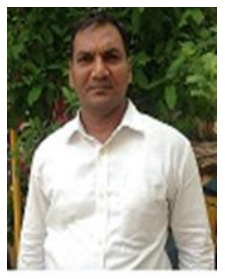

Dr. Ch Ramesh Babu received his Ph.D and M.Tech in CSE from JNTUK Kakinada and B.Tech in CSE from JNTU Hyderabad. Currently he works as a Professor in the Department of Computer Science and Engineering in GCET, Cheeryal, Hyderabad. He has 16 years of teaching and research experience. He was a reviewer for various International Journals and Conferences. His research interests include Grid Computing, Cloud Computing, Checkpointing, Image Processing, Computer Vision and Data Mining. Email:crb.challagundla@gmail.com

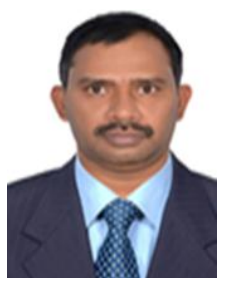

Dr. Srinivasa Rao Dammavalam received his Ph.D in CSE from JNTUK Kakinada and M. Tech in Information Technology from JNTU, Hyderabad and B.Tech in CSE from SVHCE, ANU. He worked as a research fellow at AVIRES Lab, University of Udine, Italy for two years. Currently he is working as an Associate Professor in the Department of Information Technology in VNRVJIET, Hyderabad. He has 15 years of teaching experience and 12 years of research experience. He was the recipient of the AICTE Grant for his proposal under Research Promotion Scheme for the period of two years. He was a reviewer for various International Journals and Conferences. His research interests include Image Processing, Computer Vision and Data Mining.

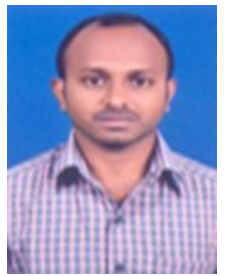

Ravi Tene received $M$. Tech in CS from JNTU, Hyderabad and B.Tech in IT from JNTU Hyderabad. Currently he is working as an Assistant Professor in the Department of Information Technology in VNRVJIET, Hyderabad. He has 11 years of teaching experience. His research interests include Image Processing and Computer Networks.

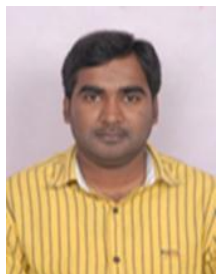

Gumma Gopi received $M$. Tech in Computers from College of Engineering Pune and B.Tech in IT from Sree Vidyanikethan Engineering College. Currently working as a Q/A Engineer at Akamai Technologies, Bengaluru, Karnataka, India. 
International Journal of Advanced Technology and Engineering Exploration, Vol 5(40)

Table1 assessment metrics for fused images obtained

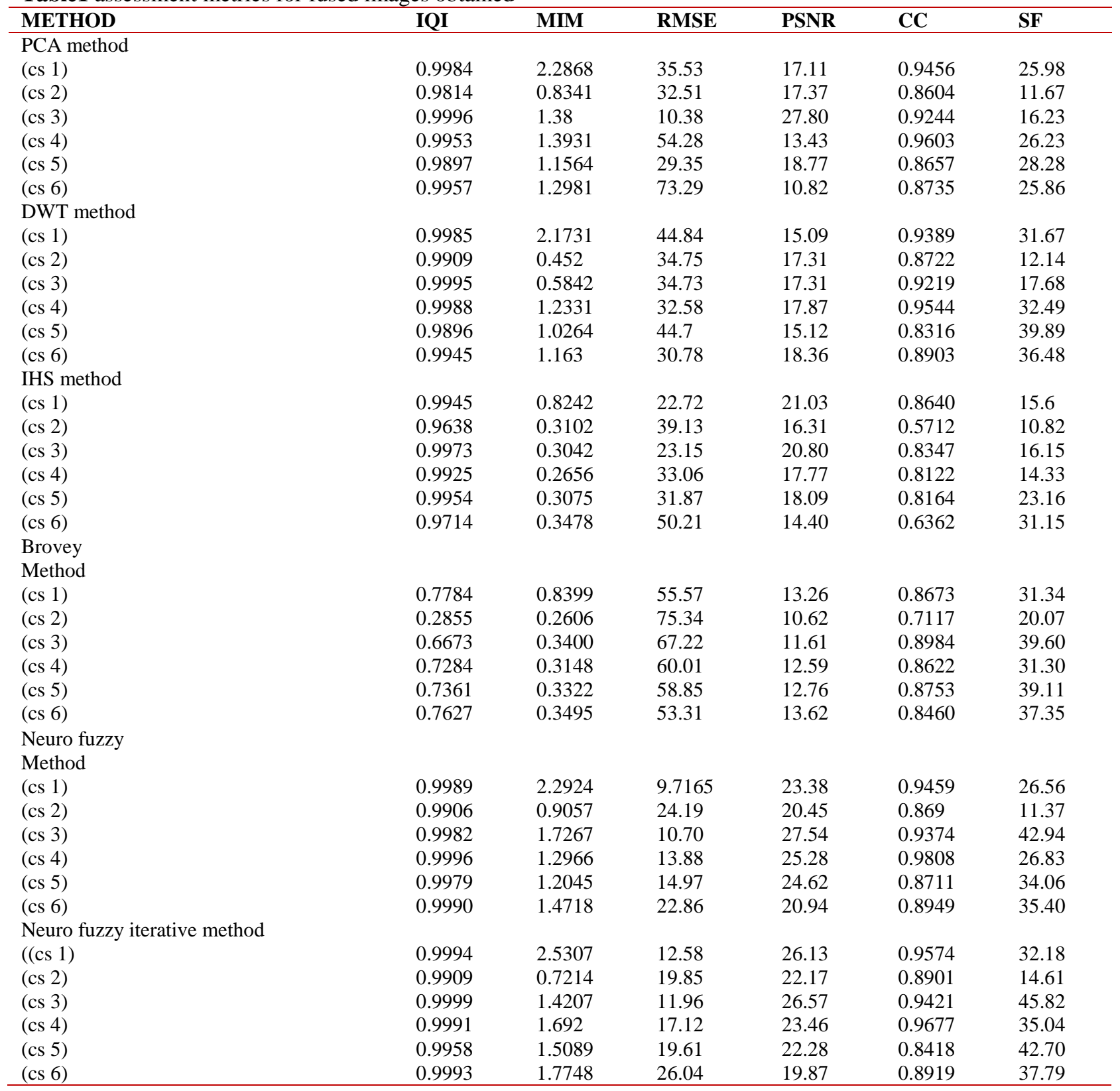

